
allemande

\title{
Sortir de la guerre avec Machiavel
}

Hans Delbrück, Otto Baumgarten et la révolte des modérés contre l'ultranationalisme au tournant de l'année 1917

\section{Christian E. Roques}

\section{(2) OpenEdition}

1 Journals

Édition électronique

URL : https://journals.openedition.org/allemagne/569

DOI : $10.4000 /$ allemagne.569

ISSN : 2605-7913

Éditeur

Société d'études allemandes

\section{Édition imprimée}

Date de publication : 29 décembre 2017

Pagination : 285-300

ISSN : 0035-0974

\section{Référence électronique}

Christian E. Roques, "Sortir de la guerre avec Machiavel », Revue d'Allemagne et des pays de langue allemande [En ligne], 49-2 | 2017, mis en ligne le 29 décembre 2018, consulté le 19 mai 2021. URL http://journals.openedition.org/allemagne/569 ; DOI : https://doi.org/10.4000/allemagne.569 


\section{Sortir de la guerre avec Machiavel \\ Hans Delbrück, Otto Baumgarten et la révolte des modérés contre l'ultranationalisme au tournant de l'année 1917}

\section{- Christian E. Roques *}

Si l'entrée en guerre des Américains en avril 1917 marque clairement un tournant dans la Première Guerre mondiale au plan international, la «crise de juillet » et la chute du chancelier Bethmann-Hollweg manifestent, parallèlement, une période de bouleversements internes à l'Allemagne. Toutefois, dans la discussion sur les buts de guerre qui fait rage depuis le début de la guerre (et qui se trouve renforcée par l'échec de l'offensive sous-marine de février 1917), la résolution de paix adoptée par le Reichstag le 19 juillet apparaît comme un moment paradoxal: alors même que l'évocation, pour la première fois, d'une "paix de compromis " (Verständigungsfrieden) semble indiquer que cette option s'impose désormais, les partisans de longue date d'une telle paix sont en perte d'influence auprès du gouvernement. C'est précisément ce qu'avait mis en évidence la décision de retour à la guerre sous-marine

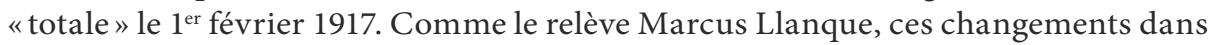
la configuration politique allemande induisent, parallèlement, un «tournant de l'argumentation politique» et un ajustement des stratégies discursives des différents acteurs $^{(1)}$.

Le présent article reprend l'idée du «tournant» en se concentrant sur ce que l'on pourrait qualifier dans ce contexte de «révolte de modérés»: face à la dégradation de la situation diplomatique et militaire, certains réseaux politiques conservateurs ou centristes, qui jusqu'alors soutenaient activement la politique du gouvernement Bethmann-Hollweg, décident de se mobiliser contre ce qu'ils considèrent comme les effets

* Maître de conférences en études germaniques (civilisation allemande) à l'Université de Reims Champagne Ardennes, CIRLEP, Reims / Groupe de Recherche sur la Culture de Weimar, FMSH Paris.

1 Marcus Llanque, Demokratisches Denken im Kriege. Die deutsche Debatte im Ersten Weltkrieg, Berlin, Akademie, 2000, p. 192 sq. Sauf indication contraire, toutes les traductions de l'allemand dans le présent article sont de notre fait. 
néfastes des outrances du mouvement nationaliste extrémiste ${ }^{(2)}$. Au cœur de cette offensive se trouvent les cercles politiques qui s'organisent autour de Hans Delbrück, historien de renom et éditeur influent des Preußische Jahrbücher. Il semblait donc pertinent de s'intéresser aux stratégies discursives mises en place par ce réseau pour contrecarrer la propagande ultranationaliste et faire avancer auprès du grand public, ou du moins du public bourgeois lettré, la cause de la paix négociée. Dans cette optique, nous nous proposons de consacrer une attention particulière à la principale plume de l'offensive éditoriale organisée par Delbrück: le théologien Otto Baumgarten. Son cas mérite d'être retenu, non seulement parce qu'il sera l'auteur d'un texte de dénonciation de l'extrémisme nationaliste largement diffusé, mais également parce que, l'année précédente, il publie un livre de réflexion sur le rapport entre «morale et politique» ${ }^{(3)}$ qui expose le fondement théorique de l'offensive antinationaliste. S'appuyant sur la référence à Machiavel, il défend l'idée d'une séparation radicale entre morale et politique, qui, selon lui, permettrait seule de revenir à une considération réaliste de la situation politique, condition nécessaire pour pouvoir sortir du conflit.

Dès lors, nous nous attacherons à présenter succinctement, dans un premier temps, Hans Delbrück et les réseaux politiques qui se structurent autour de lui dans cette mobilisation de l'année 1917, pour en montrer non seulement la cohérence politique générale mais également l'imbrication personnelle. Sur cet arrière-fond, les prises de positions d'Otto Baumgarten pourront ensuite être lues comme les interventions d'un intellectuel engagé tout autant que l'expression des convictions de tout le réseau «Delbrück». Dès lors, il deviendra possible de saisir la dimension offensive de l'argumentation développée par Baumgarten dans sa réflexion philosophique. Et dans cette optique, il s'agira, après avoir replacé l'intervention de Baumgarten dans le cadre plus général du débat sur «morale et politique» que génère le déclenchement de la guerre, d'exposer plus précisément la structure de l'argumentation développée, notamment pour y mettre en évidence la généalogie très "germanique» dans laquelle Machiavel est placé et lu. Ce n'est qu'avec une telle mise en perspective que l'on pourra, dans un troisième moment, se consacrer à l'analyse de l'argumentation du libelle sur «l'écho du mouvement alldeutsch aux États-Unis » publié en $1917^{(4)}$ pour en mettre en avant les traits pamphlétaires et militants.

Si Hans Delbrück est aujourd'hui bien moins connu que certains de ses collègues historiens de la même période, comme Ernst Troeltsch ou Friedrich Meinecke, il n'en fut pas moins une des figures importantes de la vie intellectuelle de l'Empire puis des premières années de la République de Weimar. Son profil particulier tient à son double engagement simultané comme universitaire et comme militant politique:

2 Le Alldeutscher Verband n'en est que la plus grande et la plus active des organisations. C'est pourquoi nous préférerons parler dans les pages qui suivent des «ultranationalistes» ou des «nationalistes extrémistes» pour désigner ainsi plus globalement l'ensemble de la mouvance völkisch. Cf. Rainer Hering, Konstruierte Nation. Der Alldeutsche Verband 1890 bis 1939, Hambourg, Christians, 2003, et plus généralement Uwe Puschner, Walter Schmitz, Justus H. Ulbricht (dir.), Handbuch zur "völkischen Bewegung” 1871-1918, Munich, Saur, 1996. 
«On pourrait voir en lui le dernier de cet aréopage d'historiens politiques, qui de Dahlmann à Treitschke, surent allier une activité de publiciste national-politique avec une recherche qui fut scientifique, tout en tenant aussi compte des réalités de la vie ${ }^{(5)}$.

Dans l'arène politique, l'engagement de Delbrück fut de nature libérale-conservatrice avec un fort accent patriotique, notamment comme député pour le parti conservateur libre (Freikonservative Partei) ${ }^{(6)}$. Toutefois, ce ne fut pas son activité partisane mais son rôle d'éditeur des Preußische Jahrbücher qui firent de lui un acteur politique influent. Ce journal fondé en 1858 par Rudolf Heym était devenu un «forum de discussion, dans lequel l'élite intellectuelle de l'époque pouvait prendre la parole » ${ }^{(7)}$. Seul maître à bord après la mise à l'écart de Heinrich von Treitschke en 1889, Delbrück fit des Jahrbücher «une des plus importantes revues intellectuelles d'Allemagne», notamment en y assurant chaque mois la rubrique «Correspondance politique» (Politische Korrespondenz), dans laquelle il «accompagnait les événements de l'actualité avec une critique minutieusement pondérée ${ }^{(8)}$ et devint "un des commentateurs et critiques les plus intéressants de l'époque wilhelminienne " ${ }^{(9)}$. Grâce à la revue, Delbrück disposait d'un auditoire important - non pas tant en termes de quantité que de qualité du public: elle jouissait d'une bonne diffusion au sein de l'élite intellectuelle et politique de Prusse ${ }^{(10)}$.

Sur le plan universitaire, Delbrück dut sa renommée au fait d'avoir révolutionné l'histoire militaire en l'établissant comme champ d'investigation légitime pour les historiens universitaires ${ }^{(11)}$. Chemin faisant, il remit en cause un certain nombre d'idées reçues en matière de stratégie militaire, ce qui le plaça, à partir de 1878, au cœur de la «dispute sur la stratégie» (Strategie-Streit) et le fit entrer en conflit frontal avec l'État-major de l'armée prussienne ${ }^{(12)}$, car les analyses de Delbrück débouchaient nécessairement sur une critique du «Schlieffenplan», c'est-à-dire du plan opérationnel de l'Armée allemande en cas de nouveau conflit militaire ${ }^{(13)}$. Dès lors, même si la

5 Friedrich Meinecke, «Vermischtes», Historische Zeitschrift, $140 / 3$ (1929), p. 703. Voir également Johannes Ziekursch, «Delbrück, Hans G. L.», in : Deutsches Biographisches Jahrbuch, vol. XI: «Das Jahr 1929», Stuttgart/Berlin, Deutsche Verlagsanstalt, 1932, p. 93.

6 J. Ziekursch, «Delbrück, Hans G. L.» (note 5), p. 93.

7 Joachim Kirchner, Das deutsche Zeitschriftenwesen. Seine Geschichte und seine Probleme, Wiesbaden, Otto Harrassowitz, 1962, p. 165.

8 Friedrich Carl Scheibe, «Marne und Gorlice. Zur Kriegsdeutung Hans Delbrücks», Militärgeschichtliche Mitteilungen, 53 / 2 (1994), p. 355.

9 Wilhelm DeIst, «Hans Delbrück. Militärhistoriker und Publizist», Militärgeschichtliche Mitteilungen, $57 / 2$ (1998), p. 371.

10 Annelise Тнимме, Hans Delbrück als Kritiker der wilhelminischen Epoche, Düsseldorf, Droste, 1955, p. 12. Thimme cite Kürschners Handbuch der Presse de 1902, disant que la revue est lue par les «meilleurs cercles» (beste Kreise).

11 Sven Lange, «Hans Delbrück. Der Kritiker der Kriegsgeschichte», in: Hans Ehlert (dir.), Deutsche Militärhistoriker von Hans Delbrück bis Andreas Hillgruber, Potsdam, Militärgeschichtliches Forschungsamt, 2010, p. 9.

12 Cf. Sven Lange, Hans Delbrück und der "Strategiestreit". Kriegsführung und Kriegsgeschichte in der Kontroverse 1879-1914, Fribourg-en-Brisgau, Rombach, 1995; Markus Pöhlmann, Kriegsgeschichte und Geschichtspolitik: der Erste Weltkrieg. Die amtliche deutsche Militärgeschichtsschreibung 19141956, Paderborn, Ferdinand Schöningh, 2002.

13 Hew Strachan, «1914-1918 et la redéfinition de la guerre», Politique étrangère, nº 1 (2014), p. 74. 
polémique s'atténue avant la Première Guerre mondiale, Delbrück gardera toujours, aux yeux des militaires, l'image d'un critique de l'État-major allemand ${ }^{(14)}$. Cela est d'autant plus important que s'enclenche, au tournant du siècle, une dynamique de rapprochement entre l'État-major et les mouvements d'extrême droite ${ }^{(15)}$, qui doit ellemême être replacée dans le contexte général de l'émergence, après 1871, d'une opinion publique nouvelle, très militariste et radicalement nationaliste, voire raciste ${ }^{(16)}$.

Toutefois, il s'agit de ne pas simplifier indûment les oppositions politiques de ces années, mais d'en saisir avec nuances les enchevêtrements idéologiques. Ainsi, il faut prendre conscience de la nature stratégique (plus qu'idéologique) d'un certain nombre d'oppositions pour saisir avec précision les réalités politiques de l'époque, à l'exemple de la "pétition des intellectuels » du 8 juin 1915 et de la contre-pétition du 9 juillet ${ }^{(17)}$. Si ces pétitions expriment d'évidence une nouvelle polarisation de l'espace politique, elles ne doivent pas cacher la perméabilité des oppositions:

«Les personnalités et les groupes, qui refusaient absolument d'être assimilés aux ultranationalistes et qui se décrivaient eux-mêmes comme libéraux, se retrouvaient, concernant les buts en matière de politique extérieure, d'accord avec les objectifs des ultranationalistes » ${ }^{(18)}$.

Un «libéral» comme Delbrück pouvait être politiquement plus proche d'un impérialiste comme Reinhold Seeberg que des militants pacifistes, dont il est pourtant l'allié de circonstances en $1915^{(19)}$. Dès lors, la ligne de fracture traverse d'abord le camp national lui-même:

14 Cf. Friedrich von Bernhardi, qui, dans ses mémoires en 1927, présente Delbrück comme le chef de file d'une «cabbale de savants civils» (Klüngel von Zivilgelehrten) «dont le seul plaisir consistait à pouvoir se confronter à l'armée» (cf. F. v. Bernhard, Denkwürdigkeiten aus meinem Leben, Berlin, Mittler, 1927, p. 133).

15 M. Pöhlmann, Kriegsgeschichte und Geschichtspolitik (note 12), p. 47.

16 Cf. Wolfgang J. Mommsen, «Außenpolitik und öffentliche Meinung im Wilhelminischen Deutschland 1897-1914», in: ID., Der autoritäre Nationalstaat. Verfassung, Gesellschaft und Kultur im deutschen Kaiserreich, Francfort-sur-le-Main, 1990, p. 358-379; Stig Förster, Der doppelte Militarismus. Die deutsche Heeresrüstungspolitik zwischen Status-Quo-Sicherung und Aggression 1890-1913, Wiesbaden, Franz Steiner, 1985.

17 Voir Klaus Schwabe, Wissenschaft und Kriegsmoral. Die deutschen Hochschullehrer und die politischen Grundfragen des Ersten Weltkrieges, Göttingen, Musterschmidt, 1969; Herbert DöRING, Der Weimarer Kreis. Studien zum politischen Bewußtsein verfassungstreuer Hochschullehrer in der Weimarer Republik, Meisenheim am Glan, Anton Hain, 1975; Steffen Bruendel, Volksgemeinschaft oder Volksstaat. Die 'Ideen von 1914' und die Neuordnung Deutschlands im Ersten Weltkrieg, Berlin, Akademieverlag, 2003.

18 Fritz Fischer, Griff nach der Weltmacht. Die Kriegszielpolitik des kaiserlichen Deutschlands 1914-1918, Düsseldorf, Droste, 1964, p. 187. Christian Jansen relève que durant l'été 1915, Delbrück et Alfred Weber cherchent à rapprocher les «impérialistes libéraux» et les «annexionnistes » autour de Seeberg. Cf. Christian Jansen, Professoren und Politik. Politisches Denken und Handeln der Heidelberger Hochschullehrer 1914-1935, Göttingen, Vandenhoeck \& Ruprecht, p. 137.

19 Cf. H. Döring, Der Weimarer Kreis (note 17), p. 29. Annelise Thimme relève que Delbrück était partisan d'une "politique de puissance mondiale» (Weltmachtpolitik) et qu'il soutint fermement la politique d'armement, notamment de construction navale (écrivant même en mars 1897 dans les Preußische Jahrbücher que «l'avenir du peuple allemand» en dépendait), du moins jusqu'en 1912. Cf. А. Thimme, Hans Delbrück als Kritiker (note 10), p. 102 et 109. 
«S’organise autour de Delbrück un cercle agissant parfois de manière conspirative, qui essayait de combattre la démagogie ultranationaliste avec ses propres armes, non parce que ses membres auraient été opposés au maintien de l'État autoritaire mais parce qu'ils en étaient venus à considérer l'activité des ultranationalistes comme le principal danger pour l'État autoritaire » ${ }^{(20)}$.

Autrement dit, le réseau social qui se structure autour de Hans Delbrück dès la fin du mois d'août 1914 (notamment à travers les fameuses «soirées du mercredi») ${ }^{(21)}$ ne regroupait nullement des anti-bellicistes, mais des patriotes convaincus du bien-fondé de la guerre menée. Toutefois, en tant que "patriotes» leur horizon premier n'était pas la victoire militaire, mais le maintien de l'État allemand. Ce qui distinguait les deux pétitions de l'été 1915 était donc bien d'abord de nature stratégique. D’une part, l'opposition entre une «paix de compromis" (Verständigungsfrieden) et une "paix victorieuse » (Siegfrieden) ${ }^{(22)}$ et, de l'autre, un désaccord quant à l'acteur politique qui doit être au cœur de la résolution du conflit: «Le chancelier, dont le maintien était l'objectif du groupe autour de Delbrück, était le symbole du primat de la politique par rapport aux intérêts de l'industrie et de l'appareil militaire " ${ }^{(23)}$.

Cependant, c'est un autre enjeu stratégique de la contre-pétition qu'évoque Delbrück dans une lettre à son ami Max Lenz:

«Évidemment, il eût été préférable que les deux pétitions ne voient pas le jour, mais dès l'instant où les autres avaient proclamé à la face du monde ce produit de la bêtise et de la brutalité, pouvions-nous laisser le peuple allemand encaisser l'insulte que ce soit cela qui paraisse incarner la voix du peuple allemand?» ${ }^{(24)}$

Avec la fin de l'année 1915, l'enjeu prioritaire devient ainsi de contrer les effets négatifs de la propagande ultranationaliste. C'est pourquoi Delbrück soutiendra activement le projet de contre-mobilisation que lui soumettent, au même moment, Martin Hobohm, un de ses anciens doctorants, et Paul Rohrbach, avec lequel Delbrück avait travaillé au sein du "Evangelisch-sozialer Kongreß».

Dès mai 1915, Paul Rohrbach, théologien protestant qui s'était illustré, en 1912, dans son livre à succès Der deutsche Gedanken in der Welt ${ }^{(25)}$ par son militantisme en faveur d'un «impérialisme allemand», avait commencé à organiser une réponse structurée aux ultranationalistes ${ }^{(26)}$. En tant que directeur du service de presse de la Zentralstelle für Auslandsdienst, le service du ministère des Affaires étrangères en charge de la propagande officielle de l'Allemagne à l'étranger, Rohrbach avait ordonné la collecte de matériau concernant l'effet de la propagande des nationalistes extrémistes à l'étranger. Grâce à l'entremise de Delbrück auprès du gouvernement,

20 M. Llanque, Demokratisches Denken im Kriege (note 1), p. 141.

21 Cf. Paul Rühlmann, «Delbrücks Mittwochabend», in: Emil Daniels, Paul Rühlmann (dir.), Am Webstuhl der Zeit. Erinnerungsgabe Hans Delbrück dem Achtzigjährigen von Freunden und Schülern dargebracht, Berlin, Reimar Hobbing, 1928.

22 Cf. Harmut Lehmann, Transformationen der Religion in der Neuzeit. Beispiele aus der Geschichte des Protestantismus, Göttingen, Vandenhoeck \& Ruprecht, 2007, p. 265.

23 S. BRUENDEL, Volksgemeinschaft oder Volksstaat (note 17), p. 80.

24 Cité par H. DöRING, Der Weimarer Kreis (note 17), p. 33.

25 Cf. F. Fischer, Griff nach der Weltmacht (note 18), p. 187.

26 Sur la création du «bureau Hobohm», cf. H. DöRING, Der Weimarer Kreis (note 17), p. 37 sq. 
ce fut l'historien Martin Hobohm qui se trouva chargé d'organiser et de gérer cette $_{\text {collecte }}{ }^{(27)}$. Face aux tergiversations du gouvernement quant à l'opportunité d'exploiter le matériau accumulé ${ }^{(28)}$, Rohrbach et Hobohm décident, à partir de juin 1916, de créer un «bureau» autonome dont l'objet serait de dénoncer la nature outrancière des ultranationalistes et ainsi d'en dévoiler la nature pseudo-patriotique $^{(29)}$. Au plan organisationnel Delbrück, Hobohm et Rohrbach sont rapidement rejoints par l'historien Walter Goetz et le théologien Otto Baumgarten, auxquels s'ajoutent progressivement August Stein, journaliste à la Frankfurter Zeitung, Ernst Jäkh, Martin Wenck et Ernst Troeltsch, ainsi que trois membres de la Zentralstelle et l'amiral Oskar von Truppel ${ }^{(30)}$. La composition de ce groupe donne un bon aperçu des groupes sociaux qui s'organisent à travers le cercle «Delbrück»: au premier chef des universitaires fidèles au gouvernement de Bethmann-Hollweg, soutenus par des publicistes que l'on peut rattacher à l'«impérialisme culturel» et certaines personnalités de l'armée, de l'industrie ou de l'administration opposées à l'isolationnisme économique des ultranationalistes.

«C'était une partie de la grande bourgeoisie et de la bourgeoisie lettrée qui s’engagea dans le combat contre le chauvinisme dominant, soutenue individuellement par quelques hauts fonctionnaires du gouvernement. Ce furent les "théologiens libéraux" qui engagèrent le combat public contre l'idéologie ultranationaliste» ${ }^{(31)}$.

Parmi eux, Otto Baumgarten joue rapidement un rôle de premier plan.

Figure importante du Bildungsbürgertum libéral et représentant militant du libéralisme social protestant dans les premières décennies du $\mathrm{XX}^{\mathrm{e}}$ siècle, Baumgarten «s'intéressait avec passion aux questions politico-historiques du passé et aux questions politico-nationales du présent ${ }^{(32)}$. En tant que fils de l'historien Hermann Baumgarten, Otto Baumgarten considérait l'engagement dans les affaires de la cité comme un héritage familial qu'il s'efforça d'honorer toute sa vie ${ }^{(33)}$.

Ordonné pasteur de l'Église protestante de Bade en 1883, Baumgarten quitte le service de l'Église au bout de quatre ans pour s'investir dans une carrière universitaire. Après une thèse à Halle sur «Herder comme prédicateur», il soutient son habilitation

27 Cf. Günter Brakelmann, Krieg und Gewissen. Otto Baumgarten als Politiker und Theologe im Ersten Weltkrieg, Göttingen, Vandenhoeck und Ruprecht, 1991, p. 64.

28 Il ne sera publié qu'en 1919: Martin Новонм, Paul Rонввасн, Die Alldeutschen, Berlin, Engelmann, 1919.

29 G. Brakelmann, Krieg und Gewissen (note 27), p. 65.

30 Les contacts personnels avec le gouvernement passaient principalement par le secrétaire d'État, Wilhelm Solf et le chef de cabinet Rudolf von Valentini (H. DörING, Der Weimarer Kreis [note 17], p. 40). Mais les relations se dégradèrent au cours de l’année 1916: dans ses mémoires Otto Baumgarten ira jusqu'à dire que Bethmann-Hollweg avait «effectivement saboté tout le travail de Hobohm» (Otto Baumgarten, Meine Lebensgeschichte, Tübingen, 1929, p. 300).

31 G. Brakelmann, Krieg und Gewissen (note 27), p. 67.

32 Ibid., p. 65.

33 On retiendra que la mère de Baumgarten est Ida von Fallersleben, dont une sœur, Helene, était la mère de Max et Alfred Weber. Le jeune Otto aura des contacts étroits avec ses cousins pendant sa jeunesse et gardera toujours une proximité amicale et politique avec ceux-ci. C'est lui qui mariera Max et Marianne Weber en 1893 à Oerlingshausen. Cf. G. Brakelmann, Krieg und Gewissen (note 27), p. 9 et 11 . 
à Berlin en 1889 avec un travail sur «la position de Herder à l'égard du rationalisme». Nommé professeur «extraordinaire» de théologie pratique à Iéna de 1890 à 1894, il est appelé à Kiel en $1894^{(34)}$.

Parallèlement, Baumgarten milite sur le terrain politique en participant à la création du «Evangelisch-sozialer Kongreß» en 1890, dont il deviendra le président entre 1912 et 1925 (et dans lequel Delbrück et son gendre Adolf von Harnack sont des éléments moteurs ${ }^{(35)}$ ). Baumgarten est alors proche d'Ernst Troeltsch, Max Weber et Friedrich Naumann. Comme publiciste, il contribue depuis sa création en 1887 à la revue Die christliche Welt, dirigée par Martin Rade, devient co-éditeur (à partir de 1892) de la Zeitschrift für praktische Theologie et dirige à partir de 1901 la Monatschrift für Kirchliche Praxis devenue Evangelische Freiheit en 1907. Enfin, l'implication militante de Baumgarten dans différents débats publics lui vaudra une indéniable notoriété: en 1896, il prend ouvertement position en soutien aux ouvriers grévistes du port de Hambourg et en 1911, il s'investit dans la défense des pasteurs Karl Jatho, puis Gottfried Traub dans le cadre des procédures disciplinaires pour «hérésie» (Irrlehre) lancées contre ceux-ci ${ }^{(36)}$. «Au moment où éclate la guerre, Baumgarten est donc un professeur de cinquante-six ans connu de l'opinion publique allemande ${ }^{(37)}$. Dénoncé comme «socialiste» par la presse conservatrice, il fait partie des «réformateurs sociaux conséquents issus de la bourgeoisie " ${ }^{(38)}$. Dans cette perspective, l'engagement de Baumgarten dans l'offensive éditoriale du «bureau Hobohm », au-delà du rapport personnel qu'il entretient avec Delbrück et son cercle proche, ne surprend guère.

Surtout, étant donné que Martin Hobohm sert d'assistant à Baumgarten à Kiel durant le semestre d'hiver $1915^{(39)}$, il semble permis de considérer que le cours que ce dernier tient au même moment et qu'il publie ensuite en 1916 sous le titre «Morale et Politique ${ }^{(40)}$ est (au moins partiellement) le résultat des discussions entre les deux hommes. C'est pourquoi nous nous permettons d'étendre l'essentiel des positions défendues pas Baumgarten à l'ensemble du «bureau Hobohm», voire au-delà au gros des troupes qui s'organisent autour de Delbrück.

Toutefois, pour saisir dans toute sa profondeur le champ historique dans lequel Baumgarten intervient, il faut contextualiser son intervention de double manière.

Premièrement, la question philosophique et éthique (très classique) du rapport entre «morale et politique», à laquelle Baumgarten s’attache dans son livre, a connu un regain d'intérêt manifeste dans le débat public allemand depuis le début de la guerre et les principaux protagonistes du débat étaient, outre Baumgarten, August Dorner ${ }^{(41)}$, Ernst

34 Ibid., p. 10 sq.

35 H. Lehmann, Transformationen der Religion (note 22), p. 258.

36 Hasko von BAssi, Otto Baumgarten. Ein moderner Theologe im Kaiserreich und in der Weimarer Republik, Francfort-sur-le-Main, Peter Lang, 1988, p. 90 sq.

37 G. Brakelmann, Krieg und Gewissen (note 27), p. 13.

38 Ibid., p. 12 sq.

39 Hans Schleier, Die bürgerliche deutsche Geschichtsschreibung der Weimarer Republik, Cologne, Pahl-Rugenstein, 1975, p. 540.

40 O. Baumgarten, Politik und Moral (note 3).

41 August Dorner, Recht, Moral und Politik mit Beziehung auf den gegenwärtigen Krieg, Stuttgart, Spemann, 1914. 
Troeltsch $^{(42)}$, Heinrich Scholz ${ }^{(43)}$, Alfred Vierkandt ${ }^{(44)}$, Friedrich Meinecke ${ }^{(45)}$, Erich Franz ${ }^{(46)}$, Franz Sawicki ${ }^{(47)}$ et Max Adler. Si l'on peut considérer que l'intervention de Baumgarten, fin 1916, marque une rupture dans cette discussion, c'est en raison de la radicalité de son propos qui amène les interventions ultérieures à se positionner par rapport à lui. Ainsi, les livres de Franz et Sawicki se présentent explicitement comme des réponses à Baumgarten, mais l'importance de son livre est également soulignée par Max Adler en 1918, qui le décrit comme "relativement bizarre et passionnant à la fois»" ${ }^{(48)}$. Construisant son livre sur une synthèse de l'ensemble du débat, Max Adler met bien en valeur la problématique générale de celui-ci et son actualité permanente depuis 1914:

"Jamais auparavant la fameuse phrase qui dit que la guerre n'est que "de la politique par d'autres moyens" ne s'est avérée aussi vraie que maintenant. Mais, dès lors, l’antagonisme entre la politique et la morale devait devenir d'autant plus tranché que la forme ultime d'expression politique dont dispose l'État se révélait dépendre seulement de sa capacité à défaire l'ennemi.»

Cette prise de conscience aurait causé «la commotion spirituelle la plus vive», notamment en Allemagne ${ }^{(49)}$. Par conséquent, la question morale débattue n'est pas celle de l'éthique individuelle, mais bien celle de la place de la morale dans le débat et l'action politique collective, la «morale de l'État» (E. Troeltsch).

Deuxièmement, si une telle interrogation explique évidemment que Baumgarten choisisse d'introduire la référence à Machiavel dans ses réflexions, on retiendra que son intérêt pour les théories politiques du secrétaire florentin et sa connaissance de ces dernières sont nourris par les échanges avec Hobohm, auteur d'une thèse monumentale sur l'art de la guerre chez Machiavel publiée juste avant la guerre ${ }^{(50)}$ et sur laquelle Delbrück lui-même s'appuiera pour son chapitre consacré à Machiavel dans son «Histoire de l'art de la guerre» publiée en $1920^{(51)}$. À quoi s'ajoute le fait qu'un autre membre actif des "soirées du mercredi» fut Friedrich Meinecke, qui sera au cœur de la réception de Machiavel dans l'immédiat après-guerre. L'idée de la référence à Machiavel et de son utilisation stratégique dans les débats politiques de la période

42 Ernst Troeltsch, «Privatmoral und Staatsmoral», Die neue Rundschau, $27 / 2$ (1916), p. 145-169, repris in: ID., Deutsche Zukunft, Berlin, S. Fischer, 1916.

43 Heinrich Scholz, Politik und Moral. Eine Untersuchung über den sittlichen Charakter der modernen Realpolitik, Gotha, Perthes, 1915.

44 Alfred Vierkandt, Machtverhältnisse und Machtmoral, Berlin, Reuther und Reichard, 1916.

45 Friedrich Meinecke, «Kultur, Machtpolitik und Militarismus», in: Otto Hintze, Friedrich Meinecke, Hermann Oncken, Hermann Schumacher (dir.), Deutschland und der Weltkrieg, Leipzig/Berlin, Teubner, 1915, p. 617-643.

46 Erich Franz, Politik und Moral. Über die Grundlagen politischer Ethik, Göttingen, Vandenhoeck \& Ruprecht, 1917.

47 Franz SAwicki, Politik und Moral, Paderborn, Ferdinand Schöningh, 1917.

48 Max Adler, Politik und Moral, Leipzig, Verlag der Naturwissenschaften, 1918, p. 46. Chez Adler, le livre de Baumgarten apparaît notamment comme la troisième grande étape de la réflexion sur le sujet pendant la guerre après les interventions de Troeltsch et de Scholz.

49 Ibid., p. 17.

50 Martin Новонм, Machiavellis Renaissance der Kriegskunst, Berlin, Curtius, 1913.

51 Hans Delbrück, Die Geschichte der Kriegskunst im Rahmen der politischen Geschichte, 4. Teil: Die Neuzeit, Berlin, Georg Stilke, 1920. 
doit donc être lue moins comme une préoccupation personnelle de Baumgarten que représentative de l'ensemble du groupe Delbrück.

Le point de départ de la réflexion de Baumgarten est le constat que «de manière répétée pendant la guerre, non seulement des gens du peuple, mais également des personnes supposées savantes mélangeaient sans discontinuer des points de vue, des indignations et des reproches relevant de la morale à leurs considérations politiques » ${ }^{(52)}$. Le sujet principal des interrogations politico-morales, notamment au début de la guerre, fut donc, selon Baumgarten, la question de la culpabilité dans le déclenchement de celle-ci. Or une telle réflexion aurait, d'une part, toujours abouti à la conclusion que la responsabilité était celle des autres, c'est-à-dire celle des forces de l'Entente, mais, d'autre part, elle aurait systématiquement débouché sur la condamnation morale de l'ennemi: celui-ci ne serait pas seulement ce que l'on combat militairement, mais aussi ce qu'il faut soumettre, voire détruire, car moralement mauvais. Or une telle entreprise serait «absurde et dommageable» ${ }^{(53)}$.

«Absurde» parce qu'elle revient à nier ce que Baumgarten avait présenté dans un article de la Kieler Zeitung, le 12 août 1914, comme le «destin allemand»: la guerre serait la conséquence nécessaire du conflit entre des puissances impériales en perte de vitesse (c'est-à-dire la France, mais aussi, voire surtout, le Royaume-Uni) et une future puissance mondiale en train d'émerger ${ }^{(54)}$. L'Allemagne ne serait donc pas engagée dans un conflit militaire en raison de la bassesse morale de ses ennemis, mais parce que son devenir historique l'y destinait, la guerre était devenue «inévitable sur la durée». Et selon Baumgarten, «il s'agit de ressentir cette dimension tragique de la grandeur ${ }^{(55)}$.

Dès lors, développer un discours politique qui ne fait qu'insister sur la déficience morale de l'ennemi (comme c'est le cas des ultranationalistes) devient «dommageable», car il rend toute sortie du conflit impossible. Il serait donc temps «de faire justice aux exigences de l'objectivité et de l'équité allemandes aussi à l'égard des ennemis » ${ }^{(56)}$. Une pensée véritablement allemande réunirait ces deux qualités et saurait faire la part entre la politique et la morale. Toute attitude contraire se trouverait en décalage avec l'esprit national allemand: «L'immixtion de l'indignation morale dans les considérations et les appréciations politiques doit s'arrêter. Elle n'est pas digne du peuple de Bismarck» ${ }^{(57)}$.

Partant de là, Baumgarten retrace la généalogie des « réalistes » germaniques, ce qui le mène de Bismarck et Treitschke à Frédéric II. Mais arrivé à ce point, il se voit forcé de constater que même ce «génie politique n'arrive pas à se défaire des lois fondamentales que le plus grand des théoriciens de la politique, Machiavel, a mises en évidence sans aucun ménagement» ${ }^{(58)}$. C’est donc chez ce Machiavel très «germanique» que

52 O. Baumgarten, Politik und Moral (note 3), p. 1.

53 Ibid., p. 1.

54 Otto Baumgarten, «Ein deutsches Schicksal», Kieler Zeitung, 14.08.1914, cité par G. Brakelmann, Krieg und Gewissen (note 27), p. 22.

56 Ibid., p. 3.

57 Ibid., p. 5.

58 Ibid., p. 9. 
Baumgarten va aller puiser «les lois fondamentales que Le Prince a données à l'action des hommes d'État, celles qu'ont respectées aussi bien Frédéric le Grand que Bismarck dans leur entreprise de création d'État et qu'il suffit de débarrasser de l'apparence d'immoralité pour parvenir à des considérations claires et satisfaisantes sur la morale et la politique ${ }^{(59)}$.

S’il ne peut s'agir de retracer ici en détail les nuances de l'argumentation historique (première partie du livre) et systématique (deuxième partie du livre) mise en place par Baumgarten, il faut souligner le rôle crucial conféré à la référence à Machiavel. Trois caractéristiques de cette réception doivent ici être retenues.

Nous venons de mentionner la première: Baumgarten lit Machiavel comme l'inspirateur de la tradition allemande du réalisme politique ${ }^{(60)}$. Son origine italienne n'entre donc pas en jeu: au contraire, Baumgarten explique que le contexte historique immédiat qui permettrait d'expliquer certains traits de la doctrine machiavélienne ne l'intéresse pas du tout ${ }^{(61)}$. C'est bien sa «modernité » qui l'intéresse et qu'il applaudit:

«Son mérite est la redécouverte et l'approfondissement de la véritable méthode des hommes d'État, la mise au jour scientifique des lois particulières sur lesquelles repose l'art politique moderne» ${ }^{(62)}$.

Cela met en évidence le deuxième trait de cette réception: sa nature stratégique. Le texte de Baumgarten n'est pas un essai universitaire sur la doctrine machiavélienne qui s'appuierait sur une connaissance précise du texte originel. Les pages consacrées à Machiavel reposent sur une source principale et quasi unique qu'est Martin Hobohm (explicitement cité). S'il renvoie également aux textes de son propre père, l'historien Hermann Baumgarten (qui a consacré un chapitre à Machiavel dans sa monumentale biographie sur Charles Quint ${ }^{(63)}$ ), c'est en revanche pour en rejeter la conception par trop négative. Et alors qu'il rejette les arguments historiques pour éclairer la pensée politique du Florentin, il y recourt pour défendre ce dernier contre le reproche d'antihumanisme:

«Pourtant, je reste convaincu, avec Hobohm, de pouvoir trouver chez Machiavel des vérités essentielles. Treitschke montre dans sa "Politique" comment la part d'ombre soulignée par mon père s'explique en grande partie par cette époque de transition, dans laquelle Le Prince fut écrit» ${ }^{(64)}$.

Dès lors, si la référence à Machiavel est bien de type stratégique, la question qui se pose est, pour finir, celle de l'objectif de cette utilisation: affirmant s'appuyer sur la

59 Ibid., p. 10.

60 Une position qui fait écho à celle d'Enrico Ruta, le jeune philosophe que Benedeto Croce charge en 1916 de traduire Politik de Heinrich von Treitschke. Dans la préface à la traduction publiée deux ans plus tard Ruta note avec une «satisfaction patriotique» que «le fondement sur lequel Treitschke fonde sa science de l'État est notre conception italienne, c.-à-d. celle de Machiavel, à savoir celle de l'État compris comme puissance». Cf. Frederico Trocini, «Machiavellismus, Realpolitik und Machtpolitik. Der Streit um das Erbe Machiavellis in der deutschen politischen Kultur der zweiten Hälfte des 19. Jahrhunderts», in: Cornel Zwierlein, Annette Meyer (dir.), Machiavellismus in Deutschland. Chiffre von Kontingenz, Herrschaft und Empirismus in der Neuzeit, Munich, Oldenbourg, 2010, p. 215. O. Baumgarten, Politik und Moral (note 3), p. 54.

62 Ibid., p. 50.

63 Hermann Baumgarten, Geschichte Karls V., Stuttgart, Cotta, 1885-1892.

O. Baumgarten, Politik und Moral (note 3), p. 55. 
pensée du secrétaire florentin, Baumgarten va introduire une séparation radicale entre la sphère politique et la sphère morale; l'idée que "pour l'homme politique la morale est toujours au service du succès politique, elle n'est jamais le but ultime, elle n'est que moyen pour une fin, l'affirmation et le maintien de l'État " ${ }^{(65)}$. Ce que permettrait de penser Machiavel, c'est qu'au-delà de la morale individuelle, l'État est soumis à sa morale propre, régie par un principe unique: assurer sa survie. C’est cette idée que Baumgarten va mettre en valeur dans le dernier chapitre de sa partie historique, qu'il consacre à Treitschke (le théoricien) et Bismarck (le praticien). La généalogie florentino-germanique ainsi proposée au lecteur, et que Baumgarten systématise ensuite de manière radicale dans la deuxième partie de son ouvrage, n'en est que plus frappante. Baumgarten argumente en faveur de ce qu'il qualifie lui-même de «relativisme politico-éthique» (politisch-sittlicher Relativismus) en soulignant que dans le champ politique «le critère est ce qui est conforme à la fin, ce qui est intelligent, ce qui est, dans un sens supérieur du mot, utile, non ce qui est bon ou ce qui est beau» ${ }^{(66)}$. Dès lors, c'est en «réaliste» revendiqué que Baumgarten peut affirmer que «la plus grande vertu de l'homme politique consiste à déplacer les personnes comme des pièces d'un jeu d'échec sur le grand échiquier des affaires de l'État " ${ }^{(67)}$.

$\mathrm{Au}$ bout du compte, cette proposition de politique totalement amorale, présentée comme un machiavélisme conséquent dans la tradition de Bismarck et Treitschke, doit être replacée dans la perspective du point de départ du livre: la dénonciation des discours nationalistes qui diabolisent l'ennemi en le condamnant moralement. Dans cette optique, il apparaît clairement que le livre de Baumgarten vise à ouvrir l'option d'un espace de négociation (sans préalables moraux) entre les ennemis actuels du conflit militaire afin de trouver les moyens de renouer la nécessaire discussion et sortir de la guerre par une paix négociée assurant ce qui vient d'être présenté comme le suprême bien politique: la survie de l'État.

C'est dans une telle perspective politique militante que le livre de Baumgarten ne constitue qu'un premier moment (de clarification théorique) dans la mobilisation de l'ensemble du groupe Delbrück contre la propagande ultranationaliste. Leur «contreoffensive» ne deviendra efficace et véritablement percutante qu'avec l'attaque frontale du brûlot anti-alldeutsche que Baumgarten publie l'année suivante.

Avec le début de l'année 1917, le «bureau Hobohm» passe finalement à l'offensive (contre l'avis du gouvernement) en publiant, dans un premier temps, la "Correspondance allemande» (Deutsche Correspondenz). Cette "lettre d'information» se compose de courts commentaires sur le chauvinisme en Allemagne et à l'étranger en grande partie rédigés par Wenck lui-même, aidé de Hobohm et de Rohrbach ${ }^{(68)}$. Elle est envoyée gratuitement à près de 150 journaux ou revues, essentiellement libéraux ${ }^{(69)}$.

Mais le cœur du projet, c'est-à-dire la publication du matériau collecté au sein de la presse étrangère pour démontrer les dégâts causés par l'extrémisme des

65 Ibid., p. 60.

66 Ibid., p. 154.

67 Ibid., p. 134.

68 G. Brakelmann, Krieg und Gewissen (note 27), p. 66.

69 H. v. BAssi, Otto Baumgarten (note 36), p. 140. 
ultranationalistes, prend forme au printemps 1917 avec la création de la collection «Der Tag der Deutschen » chez l'éditeur Diederichs. Le premier livre publié sera celui d'Otto Baumgarten sur «l'écho du mouvement ultranationaliste en Amérique» ${ }^{(70)}$. Rédigé dans l'urgence au cours des premiers mois de l'année 1917, le petit fascicule paraît le 29 mars et rencontre un indéniable succès: fin mai, les 5000 exemplaires de la première édition sont épuisés ${ }^{(71)}$.

Comme il l'explique lui-même dès les premières lignes, Baumgarten rédige son texte à un moment «où nous attendons à tout moment [...] la déclaration de guerre de l'Amérique du Nord». Or dans un tel contexte, «s'impose à nous, avec force, la question de savoir comment expliquer l'ambiance hostile du peuple américain à l'encontre du peuple allemand auquel le lie une amitié traditionnelle». Et Baumgarten de donner l'explication dans la foulée: «C'est, pour le dire en peu de mots, l'affirmation que l'esprit ultranationaliste, qui menace le monde, serait celui qui domine en Allemagne » ${ }^{(72)}$.

Le point de départ de Baumgarten semble donc être la dénonciation de l'impression erronée que la "presse jingoïste» donne de l'Allemagne, c'est-à-dire la critique du discours antiallemand du chauvinisme américain. Mais cette position «défensive» est immédiatement problématisée sur un ton ironique: ainsi, Baumgarten explique-t-il qu'il ne saurait s'agir d'excuser la malveillance américaine " par la mise en accusation de compatriotes allemands qui par leur démesure et leur exagération de la pensée allemande dans le monde auraient quasi contraint les Américains à leur parti pris contre l'être et la politique allemands " ${ }^{(73)}$. Dès lors, la véritable thèse de Baumgarten est plus offensive:

«Le mouvement ultranationaliste donne aux va-t-en-guerre américains le prétexte et la possibilité de conférer à leurs tendances affairistes et à leurs intérêts de pouvoir l'apparence d'une intervention aux nobles intentions humanitaires " ${ }^{(74)}$.

Il va même plus loin en affirmant que les propagandistes exaltés des idées ultranationalistes sont «responsables» de la diffusion, au sein de la presse américaine, des préjugés antiallemands. Et Baumgarten va documenter son propos en citant longuement des exemples de pamphlets ou d'articles de presse américains qui évoquent, voire citent eux-mêmes, des textes particulièrement choquants du mouvement ultranationaliste. L'argumentation s'organise en quatre grands champs thématiques: d'abord les éléments de discours qui «menacent l'Amérique», puis les extraits qui documentent le "pangermanisme comme la rage [Tollwut] de l'Allemagne», suivi d'un chapitre consacré à la «religion de la guerre» des ultranationalistes avant de finir avec une étude de leur «culte de la haine».

Nous nous contenterons de relever ici les éléments du livre de Baumgarten qui articulent son intention première: ouvrir un front de guerre éditoriale contre les

70 O. Baumgarten, Das Echo (note 4).

71 H. v. BAssi, Otto Baumgarten (note 36), p. 141. On retiendra que parmi les obstacles à surmonter par le «bureau Hobohm» pour la publication de la collection se trouvait le refus du gouvernement de lui attribuer un contingent de papier, ce qui obligea au rachat d'un petit éditeur afin de pouvoir disposer du contingent de celui-ci (cf. H. DörIng, Der Weimarer Kreis [note 17], p. 38).

Ibid., p. 1. 
propagandistes ultranationalistes. Cela transparaît, d'abord, dans la manière qu'a l'auteur de présenter les arguments de dénonciation de l'idéologie ultranationaliste comme évidents, incontestables. Dès lors, il multiplie les formules concessives: ainsi, «on ne saurait faire reproche aux Américains» ${ }^{(75)}$ de leurs conclusions antigermaniques, car "on ne saurait nier» ${ }^{(76)}$ que les idées qu'ils dénoncent se trouvent bien dans la propagande nationaliste extrémiste. De plus, l’argumentation de Baumgarten glisse progressivement de la citation des textes américains vers des textes que ceux-ci auraient $p u$ citer. C'est manifeste au milieu du deuxième chapitre, où il explique que «les personnes qui lisent régulièrement les Alldeutsche Blätter devront avouer que de telles envolées d'autoglorification et d'inhumanité qui confinent à de la folie n'y sont pas chose vraiment rare. Mais on trouve la même chose dans d'autres productions ultranationalistes... ${ }^{(77)}$. Et Baumgarten de citer directement le livre de Joseph Ludwig Reimer ${ }^{(78)}$ ou des articles du Volkserzieher sans passer par l'intermédiaire de citations de presse américaine ou anglaise ${ }^{(79)}$. La nature offensive de l'argumentation est également perceptible dans les compliments que Baumgarten adresse à certains auteurs américains qu'il vient de citer: ainsi quand il qualifie d' «écrivain très talentueux» le pacifiste anglais Goldworthy Lowes Dickinson et qu'il qualifie ses conclusions très antinationalistes de «fines et méritant d'être considérées » ${ }^{(80)}$. Enfin, quand l'argumentation touche au domaine de compétence particulier de Baumgarten, la théologie, avec la thématique de l'origine "germanique» du christianisme, le voile "américain » tombe totalement et l'argumentation devient pleinement celle de l'auteur. Se penchant sur le «culte germano-chrétien» (germanische-christlicher Kult), Baumgarten renvoie explicitement aux revues ultranationalistes, voire racistes que sont Heimdall, Der Volkserzieher, Der Hammer ou Ostara, et il s'amuse à tourner en ridicule l'argumentation de Jörg Lanz von Liebenfels en citant «pour rigoler un peu» ${ }^{(81)}$ les étymologies farfelues que celui-ci avance pour justifier l'idée de l'origine germanique de la culture technique.

Dans la conclusion de l'ouvrage apparaît, dès lors, l'horizon ultime de la lutte ainsi engagée aux yeux de notre auteur:

"L'influence des ultranationalistes traverse toutes nos relations avec les États-Unis comme une destinée fatale. L'on n'exagèrera sûrement pas si l'on résume l'écho que trouve le mouvement ultranationaliste en Amérique à l'éveil d'une attitude hostile à notre égard, sur laquelle les détenteurs du pouvoir peuvent s'appuyer s'ils veulent imposer la paix au monde en entrant en guerre aux côtés de l'Entente ${ }^{(82)}$.

75 Ibid., p. 10.

76 Ibid., p. 13.

77 Ibid., p. 15.

78 Joseph Ludwig Reimer, Ein pangermanistisches Deutschland. Versuch über die Konsequenzen der gegenwärtigen wissenschaftlichen Rassenbetrachtung für unsere politischen und religiösen Probleme, Berlin, Luckhardt, 1905.

79 O. Baumgarten, Das Echo (note 4), p. 15. On ne manquera de relever que dans son anglophilie Baumgarten transforme en auteur américain «Henry» Lichtenberger, le germaniste français Henri Lichtenberger, auteur d'un livre sur L’opinion américaine et la guerre (Paris, Bloud et Gay, 1915).

80 O. Baumgarten, Das Echo (note 4), p. 18.

81 Ibid., p. 25.

82 Ibid., p. 33. 
Mise en perspective avec les idées de Baumgarten sur Machiavel, une telle phrase montre qu'il ne s'agit plus ici d'une critique des effets externes de la propagande ultranationaliste, mais une dénonciation des blocages internes qu'elle provoque. Le reproche fondamental de Baumgarten est bien de nature politique: les outrances des ultranationalistes menacent le but ultime d'une politique bien comprise. Leur extrémisme porte la responsabilité directe d'un renforcement de l'Alliance ennemie en poussant les États-Unis à déclarer la guerre à l'Allemagne. Et de ce fait la paix va probablement s'imposer, non au monde, mais à la seule Allemagne, et cela sous la forme d'une "paix victorieuse» alliée qui passe nécessairement par la défaite allemande - remettant en cause la survie même de l'État. Dès lors, les vrais ennemis du pays sont ceux dont la politique empêche toute sortie victorieuse du conflit par l'établissement d'une paix négociée, et dont le seul horizon est, dès lors, la défaite totale de l'Allemagne ${ }^{(83)}$.

Il serait probablement exagéré de dire que l'offensive éditoriale des cercles «Delbrück» eut une influence cruciale sur la fin de la guerre. Mais pour autant la stratégie argumentative mise en place par ce groupe, et plus particulièrement par Baumgarten, est historiquement significative à plusieurs titres.

D'abord, dans une perspective d'histoire politique de l'Allemagne moderne, par le fait qu'à travers les cercles qui se mobilisent ainsi dans la lutte en faveur d'une paix négociée se cristallise progressivement un ensemble de personnalités qui formeront par la suite le gros des troupes de ce qu'on a coutume d'appeler les «républicains par raison». Avec Delbrück, Harnack, Troeltsch, Meinecke, Baumgarten et d'autres, il s'agit bien là des futurs partisans de la République de Weimar, dont le soutien sera crucial pour la jeune République (même s’il se révéla insuffisant à enrayer la dynamique antidémocratique) ${ }^{(84)}$.

Ensuite, dans une perspective d'histoire des idées politiques, parce que le travail de réception des théories politiques de Machiavel que lancent Delbrück, Hobohm et Baumgarten, avec l'insistance qu'ils mettent sur la séparation tranchée entre politique et morale, n'est que le premier moment d'un retour en force de Machiavel dans les années d'immédiat après-guerre. Une première vague d'intérêt pour la pensée politique du secrétaire florentin dont l'aboutissement sera le livre éminemment politique de Friedrich Meinecke, Die Idee der Staatsräson in der neueren Geschichte.

83 On pourrait presque identifier là les éléments essentiels d'une «Dolchstoßlegende» inversée. Sans aller jusque-là, il n'est pas totalement annexe que Delbrück et Hobohm seront au premier rang de ceux qui se battront contre la diffusion de cette «légende» sous la République de Weimar. Cf. H. Schleier, Die bürgerliche deutsche Geschichtsschreibung (note 39); Arden BuchHolz, Hans Delbrück and the German Military Establishment. War Images in Conflict, Iowa City, University of Iowa Press, 1985.

84 Sur le sujet, cf. H. Döring, Der Weimarer Kreis (note 17) et Andreas Wirsching, Jürgen Eder (dir.), Vernunftrepublikanismus in der Weimarer Republik. Politik, Literatur, Wissenschaft, Stuttgart, Franz Steiner, 2008. 


\title{
Résumé
}

Si la défaite allemande se dessine de plus en plus nettement avec l'entrée en guerre des USA en 1917, celle-ci est précédée en Allemagne par un sursaut des défenseurs d'une paix négociée. Dans la foulée de l'historien et éditeur des Preußische Jahrbücher, Hans Delbrück, des universitaires et des intellectuels libéraux ou conservateurs modérés se mobilisent pour dénoncer la propagande outrancière des ultranationalistes comme le Alldeutscher Verband. Au premier rang de cette mobilisation le théologien libéral Otto Baumgarten, dont Politik und Moral et Das Echo der alldeutschen Bewegung in Amerika, qu'il publie en 1916-1917, livrent la clé de la contre-offensive: les deux textes montrent comment le recours à la pensée de Machiavel permet d'argumenter en faveur d'une séparation radicale entre politique et morale afin de rejeter la condamnation morale des ennemis par la propagande extrémiste. Dès lors, Baumgarten renverse l'argument patriotique en montrant que le jusqu'au-boutisme des ultranationalistes est la véritable menace pour l'Allemagne.

\section{Zusammenfassung}

Dem Eintritt der amerikanischen Streitkräfte 1917 und der sich damit immer klarer abzeichnenden Niederlage des deutschen Reiches geht in Deutschland eine Mobilisierung der liberal-konservativen Kräfte voraus, die einen „Verständigungsfrieden“ vertreten. Angeführt durch den Historiker und Herausgeber der Preußischen Jahrbücher, Hans Delbrück, organisieren sich Professoren und Publizisten für eine Offensive gegen die alldeutsche Propaganda. An erster Front dieses Gegenangriffs: der sozialliberale Theologe Otto Baumgarten, dessen zwei, 1916-1917 veröffentlichen Bücher, Politik und Moral und Das Echo der alldeutschen Bewegung in Amerika, den argumentativen Schlüssel zur Mobilisierung liefern: durch den Rückgriff auf Machiavelli wird eine radikale Trennung von Politik und Moral argumentiert, was die von den alldeutschen betriebene moralische Verdammung der Feinde bestreiten soll. Somit kann Baumgarten dann das patriotische Argument umkehren und zeigen, dass die eigentlichen Feinde Deutschlands die Vertreter des extremistischen Nationalismus sind.

\begin{abstract}
As the USA join the allied forces against Germany in 1917, the German defeat appears more and more a real possibility. But the American intervention is preceded in Germany by the mobilization of the proponents of a negotiated peace. Led by the historian and editor of the Preußische Jahrbücher, Hans Delbrück, liberal academics and intellectuals organize an editorial counterattack to the propaganda of the nationalistic extremists. At the forefront of this fight: the liberal theologian Otto Baumgarten. The two books he publishes in 1916 and 1917, Politik und Moral und Das Echo der alldeutschen Bewegung in Amerika, give the key to the antinationalist campaign: using the reference to Machiavelli, Baumgarten argues in favor of a radical separation of politics and morals, effectively undermining the moral condemnation of the enemies that the ultranationalists use so often. Therefore, Baumgarten can reverse the patriotic argument and show that the real enemies of the German state are the unrelenting nationalists.
\end{abstract}

